\title{
Kinetics of mesoglobule formation and disintegration in solutions of thermoresponsive polymers after fast pressure jumps
}

\author{
C. M. Papadakis ${ }^{1}$, B.-J. Niebuur ${ }^{1}$, L. Chiappisi ${ }^{2}$, F. A. Jung ${ }^{1}$, X. Zhang ${ }^{1}$, A. Schulte ${ }^{3}$ \\ IPhysik-Department, Technische Universität München, Garching, Germany, \\ ${ }^{2}$ Institut Laue-Langevin, Grenoble, France, \\ ${ }^{3}$ Department of Physics, University of Central Florida, Orlando FL, U.S.A.
}

papadakis@tum.de

Polymeric nanoparticles are used in many fields, e.g. for drug delivery. Poly $(N$-isopropylacrylamide $)$ in aqueous solution forms nanoparticles ("mesoglobules") above its cloud point. The coexistence line of this system in the temperature-pressure frame is an ellipse with a maximum at $\sim 60 \mathrm{MPa}$ and $35^{\circ} \mathrm{C}$ [1]. We investigate the formation and growth of mesoglobules as well as their disintegration after rapid pressure jumps across the coexistence line, both at low (below $20 \mathrm{MPa}$ ) and high pressures (above $101 \mathrm{MPa}$ ). Time-resolved small-angle neutron scattering at instrument D11 (ILL Grenoble) gives structural information on a large range of length scales and in a time range from $50 \mathrm{~ms}$ to $\sim 1650 \mathrm{~s}$ after the jump.

Mesoglobule formation is found to be vastly different in the low- and the high-pressure regime [1-3]. In the low-pressure regime, we find that, initially, growth of the mesoglobules proceeds via diffusion-limited coalescence, but this process is later slowed down by the appearance of a dense and rigid shell from dehydrated polymers. The deeper the target pressure in the two-phase region, i.e. the further away from the coexistence line, the earlier the slowing-down sets in and hinders further growth. In contrast, in the highpressure regime, the chains stay hydrated and mobile, when the coexistence line is crossed towards the two-phase region, and the diffusion-limited coalescence proceeds without hindrance during the entire measuring time.

The disintegration of mesoglobules is studied by pressure jumps from the two-phase into the one-phase region, varying the target pressure [4]. At a target pressure close to the coexistence line, the release of single polymers from the surface of the mesoglobules is the dominating mechanism, whereas for target pressures deeper in the one-phase regime, the swelling of the mesoglobules by water prevails. The disintegration time decreases with increasing jump depth. The results point to the importance of the osmotic pressure of water.

These findings are key for the tuning of the switching process in applications of responsive polymers for transport and release purposes. The comparatively simple polymer PNIPAM serves as a model system for more complex biological macromolecules, such as cellulose or proteins.

[1] Niebuur, B.-J., Claude, K.-L., Pinzek, S., Cariker, C., Raftopoulos, K. N., Pipich, V., Appavou, M.-S., Schulte, A., Papadakis, C. M. (2017) ACS Macro Lett. 6, 1180.

[2] Niebuur, B.-J., Chiappisi, L., Zhang, X., Jung, F., Schulte, A., Papadakis, C. M. (2018) ACS Macro Lett. 7, 1155.

[3] Niebuur, B.-J., Chiappisi, L., Jung, F., Zhang, X., Schulte, A., Papadakis, C. M. (2019) Macromolecules $52,6416$.

[4] Niebuur, B.-J., Chiappisi, L., Jung, F., Zhang, X., Schulte, A., Papadakis, C. M. (2021) RSC Nanoscale, DOI: 10.1039/d1nr02859f

Keywords: thermoresponsive polymers; small-angle neutron scattering, time-resolved measurements

Financial support by Deutsche Forschungsgemeinschaft (DFG) is gratefully acknowledged (PA 771/22-1). A.S. acknowledges support from an August-Wilhelm Scheer visiting professorship at TU Munich. 